\title{
SULLA APPLICAZIONE DEL METODO DELLE EMULSIONI NUCLEARI ALLO STUDIO DELLA RADIOATTIVITÀ DELLE LAVE VESUVIANE (*)
}

\author{
M. Ferretti-Sforzini - C. Festa - F. Ippolito
}

È sembrato interessante applicare il metodo delle emulsioni nucleari allo studio della radioattività delle lave del Vesuvio. Tra le informazioni che sembravano ragrgiungibili con questo metodo erano le seguenti: a) conferma della variazione del contenuto in elementi radioattivi con la data di eruzione; $b$ ) identificazione dei centri di emissione delle sostanze alfa-emittenti; $c$ ) determinazione della esistenza dell'equilibrio radioattivo nella famiglia dell'Uranio e, nel caso in cui tale equilibrio non esista, determinazione del tempo di rottura dell'equilibrio stesso.

Le ricerche sono staté eseguite con lastre IIford C2 da $50 \mu$ esposte all'irraggriamento di sezioni sottili di lava (spessore superiore a 50 (s) appartenenti ad eruzioni di differente età.

È noto che una variazione del contenuto in elementi radioattivi con la data di eruzione è stata riscontrata da vari autori $\left({ }^{1}\right)$. Tali misure sono da mettere in relazione con quelle eseguite da Holmes $\left(^{2}\right)$ e più recentemente da Keevil $\left(^{3}\right.$ sui graniti.

L'interesse della ricerca sta nel cercare di stabilire le cause che abbiano potuto dar luogo a fenomeni quali quelli osservati, i quali non trovano riscontro nelle osservazioni anche di recente eseguite su prodotti lavici di altri vulcani come ad esempio dell'Etna $\left({ }^{\dagger}\right)$. D'altro canto la notevole difficoltà di procurarsi dirette informazioni sui complessi fenomeni della evoluzione e della differenziazione del magma somma-vesuviano, illustrati da Rittmann (") fino dal 1933, spinge ad approfondire alcun poco queste ricerche il cui interesse magmalogico e geochimico è di per sé evidente.

I risultati delle nostre ricerche confermano l'andamento crescente dell'attività con la data di eruzione. L'attività globale media dei

(*) Comunicazione presentata alla «Association Internationale de Sëismologie et Physique de l'intérieur de la Terre » nella $X$ Assemblea Generale dell' U.G.G.I. Roma 1954. 
tre campioni di lava appartenenti rispettivamente alle eruzioni del 79 d. C., 1760 , e 1872 è risultata pari a:

$8,5 \times 10^{-4}$ tracce alfa per $\mathrm{cm}^{2}$ e per sec. per la lava del $79 \mathrm{~d}$. C.

$31,5 \times 10^{-4}$ tracce alfa per $\mathrm{cm}^{2}$ e per sec. per la lava del 1760

$38,7 \times 10^{-4}$ tracce alfa per $\mathrm{cm}^{2}$ e per sec. per la lava del 1872 .

Nellipotesi che l'uranio contenuto nella lava sia in equilibrio con tutti i suoi discendenti da questi valori si desume una concentrazione di uranio pari a:

$0,85 \times 10^{-5}$ g di $U$ per g di lava per la lava del 79 d. C.

$3,02 \times 10^{-5}$ g di $U$ per g di lava per la lava del 1760

$3,71 \times 10^{-5}$ g di $U$ per g di lava per la lava del 1872.

I risultati parziali relativi al punto $b$ ) sono già stati pubblicati in una nota di Bachelet, Ferrelti-Sforzini e Ippolito (i). E stata allora esaminata una lava proveniente dalla colata di Castel Cisterna, attribuita, come è noto, alla eruzione "pliniana " del 79 d.C. Si tratta di una tefrite a leucite a struttura porfirica, con massa fondamentale olocristallina a grana fine. I fenocristalli (delle dimensioni di qualche mmi sono costituiti essenzialmente da augite e da leucite; quelli di augrite, più alblondanti e un po' più sviluppati (fino a $2.5 \mathrm{~mm}$ ), presentano generalmente una forma prismatica e contengono quasi sempre numerosi inclusi; quelli di leucite per lo più abluastanza limpidi contengono in renere pochissimi inclusi piuttosto piccoli. Va infine notata la presenza di qualche fenocristallo di olivina, completamente alterato, o quasi, in ematite e limonite. La massa fondamentale è costituita da plagioclasi (del tipo labrador-bitownitico), augite, leucite e minerali opachi (magnetite); subordinatamente si notano microliti di sanidina, olivina. biotite, orneblenda e apatite. In particolare nella sezione esaminata la biotite risulta piuttosto rara e per lo più priva di inclusi.

L'esame della distribuzione delle tracce delle particelle alfa indica anzituto che in questa lava non esistono minerali specifici di uranio o di torio, come del resto era prevediljile in base a considerazioni magmalogiche. Si è inoltre riscontrata l'esistenza di zone nelle quali si ha concentrazione di materiale non attivo e zone nelle quali il materiale attivo è invece particolarmente concentrato rispetto al 
resto della lastra. Le aree di densità minore corrispondono ai fenocristalli: ciò indica che nei fenocristalli intratellurici, formatisi nella massa magmatica prima della sua estrusione, non vi è stata una sensiljile sostituzione isomorfica di elementi radioattivi con altri di prossimo raggio ionico. Dobljiamo quindi ammettere che le sostituzioni isomorfiche di altri metalli da parte di elementi radioattivi avvengano in presenza della fase gassosa del magma, all'atto della eruzione e della successiva rapida consolidazione della lava. Questo fatto si inquadra perfetlamente col noto comportamento geochimico degli elementi radioattivi che tendono a restare nella fase gassosa, ed a dare, nel caso di completa solidificazione, minerali specifici negli stadi da pneumatolitico a idrotermale.

Le concentrazioni di particelle alfa superiori alla media sono presumibilmente da attribuire alla presenza nei minerali essenziali (in fenocristalli o in microliti del feltrol di qualche incluso (zircone, ilmenite, titanite) i quali, come $\dot{e}$ noto, sono relativamente ricchi di uranio e torio in sostituzione isomorfogena. Le zone rimanenti, con la loro distribuzione di particelle alfa abbastanza omogenea, ci autorizzano a ritenere che gli elementi radioattivi abbiano sostituito isomorficamente altri ioni di prossimo raggrio ionico più o meno in tutti i minerali della massa fondamentale; probabilmente l'uranio avà sostituito il calcio.

Per quanto rigruarda il punto c) si può osservare anzitutto che la evoluzione del magma somma-vesuviano ha probabilmente dato luogo a fenomeni geochimici selettivi, per azione delle masse magmatiche sulle rocce di contatto. In conseguenza di tali fenomeni può essersi verificata una alterazione dell'equilibrio tra gli elementi delle famiglic radioattive; alterazione che nel mutato chimismo del magma, passato da "ottavianitico" a "vesuvitico" (dal 79 al 1872 ) potreb)se trovare una ovvia spiegazione. D’altra parte nello studio di fenomeni (ii siflatta natura occorre considerare che gli elementi delle tre famiglie radioattive, che via via si formano, hamno caratteristiche geochimiche affatto diverse dall'elemento originario ( $U^{-38}, U^{23 .}$ o $\left.T h^{-32}\right)$ e possono quindi concentrarsi in particolari minerali di contatto o partecipare a processi differenziativi, essendo in tal modo sottratti al successivo sviluppo dei fenomeni della massa magmatica.

Per queste ragioni ci si è proposti di studiare se lalla misura degli spettri di energia delle particelle alfa si possono dedure infor- 
mazioni sullo stato di evoluzione delle famiglie radioattive nei materiali eruttati dal Vesuvio. Il problema riguarda unicamente gli elementi della famiglia dell'uranio, perché si può ritenere senz'altro che il torio sia in equilibrio con $i$ suoi discendenti in tutti e tre $i$ campioni di lava esaminati. Supponiamo che altrettanto si possa dire della famiglia dell'uranio dallo ionio in poi. Si tratta allora di determinare la concentrazione dell'uranio e dello ionio nel campione di lava: dai valori di queste concentrazioni si puó determinare un limite superiore del tempo al quale è avvenuta la rottura dell'equilibrio, ragionando come segue.

Supponiamo che la rottura dell'equilibrio sia avvenuta al tempo - $t$; il caso più probabile è che al tempo - $t$, in conseguenza di qualche processo geochimico, il materiale abloia perduto una certa quantità di torio e quindi di ionio. Sia $r_{1}$ la lunghezza minima misurabile al microscopio ed $r$, la proiezione orizzontale del massimo range residuo delle particelle alfa emesse da $U_{1}$ e da $U_{\text {II }}$ : se con $Z(r)$ si indica il numero di tracce per $\mathrm{cm}^{2}$ e per sec. la cui proiezione orizzontale è maggiore o uguale ad $r$ si ha:

$$
\begin{gathered}
\left.Z\left(r_{1}\right)=\psi[U]_{\mathrm{o}} J_{\iota_{\mathrm{I}}+u_{\mathrm{II}}}\left(r_{1}\right)+\psi f_{\mathrm{O}} \Gamma_{\mathrm{Io}}\right]_{\mathrm{e}} J_{\mathrm{Io}}\left(r_{1}\right) \\
Z\left(r_{2}\right)=\psi f_{\mathrm{o}}\left[_{\mathrm{Io}}\right]_{\mathrm{e}} J_{\mathrm{Io}_{\mathrm{o}}}\left(r_{\mathrm{2}}\right)
\end{gathered}
$$

dove $U$ è la permeabilità alfa del minerale, $[U]_{0}$ ¿ la concentrazione attuale dell'uranio, $\left[_{10}\right]$. la concentrazione di ionio in equilibrio con l'uranio. $J u_{1}+u_{11} \grave{\mathrm{e}}$ la funzione di distribuzione delle proiezioni orizzontali dei ranges delle particelle alfa emesse da $U_{\mathrm{I}}$ e $U_{\mathrm{Il}}, J_{\mathrm{Io}}$ ¿̀ la funzione di distriluzione delle proiezioni orizzontali dei ranges delle particelle alfa emesse dallo ionio in equilibrio con $i$ suoi discendenti, e $j_{0}$ è una funzione del tipo $1-\rho_{I_{0}}(\tau+)$ in cui $\lambda_{I_{0}} \dot{e}$ la costante di decadimento dello ionio $\mathrm{e} \tau$ un parametro magriore o uguale a zero (’̀ chiaro che quest'ultimo caso si verifica quando lo ionio è stato completamente asportato al tempo - $t$ ). Dalle [1] si può aquevolmente ricavare $f_{0}$ e quindi $\tau+t$. $\dot{L}:$ evidente che analoghe considerazioni valgono nel caso in cui la rottura dell'equilibrio si sia verificata a partire dal radio, anziché dallo ionio.

Le funzioni $J_{u_{1}}+u_{\mathrm{II}}$ sono quia state calcolate da Buttlar e Houtermans (': nella ipotesi che l'attività del minerale si ripartisca in modo omogeneo sulla superficie in esame, e che il minerale sia costituito da cristalli o molto grandi o molto piccoli rispetto al range delle particelle alfa entro il minerale stesso. I campioni di lava sono stati per- 
ciò studiati mediante integratore presso l'Istituto di Geologia Applicata della Universita di Napoli per stabilire le dimensioni medie dei cristalli in essi contenuti. Si è potuto stabilire che un buon $50 \%$ di essi ha dimensioni paragonabili al range delle particelle alfa nella lava, il che porterebbe ad escludere la possibilita di applicare il metodo di Buttlar e Houtermans.

Si può però osservare che la condizione sopra detta non è essenziale per l'applicazione del metodo, purché sia soddisfatta la condizione che il valore medio dell'attività su tutta la superficie $S$ esaminata non dipenda sensibilmente dalla profondita $z$ dello strato attivo. Si avranno tuttavia delle fluttuazioni attorno alla media dovute alle dimensioni finite dei cristalli. Queste fluttuazioni possono tuttavia essere calcolate e si può tener conto dell'errore da esse causato nel modo seguente: se diciamo $p(n, z)$ la probabilità di trovare is centri emittenti alla profondita $z$ si può scrivere:

$$
\frac{\partial p}{\partial z}=D^{2} \frac{\partial^{2} p}{\partial \Gamma^{2}}
$$

con $D^{2}=\frac{\overline{S^{2}} \gamma^{2}}{\xi}$ e $\Gamma=n S \cdot \xi \xi$ dove $\xi$ è uguale a una costante dell'ordine delle dimensioni lineari medie dei cristalli, $y$ è la concentrazione, $S$ la superficie media ed $n$ il numero dei cristalli attivi su un piano normale a $\approx$. L'integrale di [2] è:

$$
p(\Gamma, z)=\sqrt{\frac{1}{4 \pi\left|z-z_{0}\right| L^{2}}} \cdot e^{-\frac{\left(\Gamma-\Gamma_{0}\right)^{2}}{4\left|=-z_{0}\right| / J^{2}}}
$$

ed esprime la probabilità di trovare una attivita totale $\Gamma z d z$ ad una profonditic compresa fra $z \mathrm{e} z+d z$, nota la attività totale $\Gamma_{\left(, z_{0}\right.} d z_{0}$ ad una profondita compresa fra $z_{0}$ e $z_{0}+d z_{0}$. Ora si ha, usando i soliti simboli e seguendo il metodo di Buttlar c Houtermans:

$$
Z\left(r_{1}\right)=A \int_{r_{1}}^{\infty} d r \int_{0}^{\infty} P(r, z) \Gamma d z
$$

dove $r_{1}$ è la minima proiezione orizzontale misurata, $A$ è una costante e $P(r, z)$ la probabilita che alla profondita $z$ si abbia una proiezione orizzontale del range residuo uguale ad $r$. Tenuto conto della [3] si 
può allora dimostrare ele l'errore qualratico medio relativo su $Z\left(r_{1}\right)$ risulta :

$$
\frac{\sqrt{\overline{\Delta \prime^{2}\left(r_{1}\right)}}}{Z\left(r_{1}\right)}=\frac{\sqrt{2\left|z-z_{0}\right|}}{\sqrt{l_{0} n}}
$$

dove $z-z$ è dellordine del range', e quindi dello stesso ordine delle dimensioni lineari medie, $l_{0}$ dei cristalli. Essendo $\bar{n}$, numero medio dei cristalli attivi, molto grande, come risulta dall'esame mineralogico eseguito, l'errore su $Z\left(r_{1}\right)$ risulta minore dell'errore statistico dovuto alle nostre condizioni sperimentali e quindi può essere trascurato.

La studio del problema dellequilibrio richiede però la eliminazione delle tracce dovute al torio eventualmente presente nella lava. Ora il metodo delle emulsioni fotografiche risulta assai poco sensibile per la determinazione del rapporto dei contenuti in uranio e torio. Infatti se si contano $10^{1}$ trace per campione di lava esaminato e non si trovano tracce con proiezione orizzontale maygione di 39 $\mu$ si può tutt'al più concludere che il rapporto numero tracce Th/ numero tracce U non è superiore a circa il $10 \%$. Essendo poi a parità di concentrazione l'attività del torio circa $1 / 3$ di quella dell'uranio, se ne potrà dedurre che, in tal caso, il rapporto concentrazione Th/ concentrazione $\mathrm{U}$ non supera verosimilmente il $30 \%$, risultato assai modesto data la necessita di contare almeno $] 0^{1}$ tracee.

Ammesso però di conoscere il rapporto $T h / U$ per altre vie e con precisione migliore, si potrelhe applicare rantaggiosamente il metodo delle emulsioni nucleari allo studio dellequilibrio radioattivo perche, come si è detto, lo studio di questo problema è ricondotto alla determinazione del rapporto $l o / U$ e la situazione dal punto di vista statistico è in questo caso molto più favorevole. Occorre però tener pre. sente che, se nell'interno della lava si verifica una diffusione non trascuralibile del radon, l'equilibrio potreblo essere rotto per questa ragrone e sarelibe necessario tenerne conto. Determinazioni sperimentali per asoodare l'entiti di questa correzione sono tuttora in corso.

Vogliamo vivamente ringraziare il Prof. E. Medi, Direttore dell'Istituto Nazionale di Geofisiea, per arer messo a no:tral disposizione i mezzi necessari a questa ricerca.

Istituto di Fisica dell Lniversità, Roma - Istituto Nazionale di Geofisica, Roma - Istituto di Geologia Applicata dell Università, Napoli - Luglio 1954. 


\section{RIASSUNTO}

Il metodo delle emulsioni nucleari è stato applicato allo studio delle lave vesuviane. In particolare, per quanto riguarda il problema dellesistenza o meno dell'equilibrio fra gli elementi della famiglia dell'uranio presente nelle lave, un esame più approfondito della que. stione ha mostrato che, per ottenere informazioni sufficientemente attendibili sul rapporto torio uranio, è necessurio ridurre gli errori statistici ad una misura molto più piccola di quella generalmente ammessa dagli AA. che hanno lavorato in questo campo e si è cosi stabilito che non si potevano contare meno di diecimila tracce per campione esaminato. Occorre però tener presente che, se nell'interno della lava avviene una diffusione non trascurabile del radon, l'equilibrio potreble rompersi per questa ragione. Determinazioni sperimentali per assodare l'entità di questa correzione sono tuttora in corso.

\section{SUMMARY}

The method of the nuclear emulsion has been applied to the study. of the Vesucian lavas. In particular we hoped to get the folloning informations: a) whether the variation of the radioactive content of such lavas with the date of the eruption atas confirmed; bi whether it was possible to localize the alpha-emitters centers; ci whether it atas possible to find a method for deciding the existence of the radioactive equilibrium between the elements of the U-family inside the lavas, and, if not, to determine a superior limit for the time of rupture.

For the solution of the problem ct tee have seen that it is important to have statistical errors smaller than those generally admitted by other Authors, in order to get the value of the ratio $\mathrm{Th} / \mathrm{U}$ with a reasonable good approximation. Experimentals controls in order to decide whether a possible Rn-diffusion through surface layers of the leves should be responsible for a rupture of the equilibrium are still in progress.

\section{BIBLIOGR.AFIA}

(1) Jotx J.: "Phil. Mag. ", 18, 576 (1909). Iubò G. e Casertavo L., "Amn. Geof," 6, 315 (1953).

(2) Gutwanc, B.: Internal Constitution of the Earth. N. York (1951). p. 15t.

(3) U.G.G.I., Comité pour l'étude de la physique de lintérieur de la terre. 
Comptes rendus des séances tenues ì Bruxelles du 16 au 29 août 1951: "Ann. Géoph. "), 8, 112 (1952).

(4) Barbera L., Curatolo M., Addaho Indovina M. M., Palumbo D. e SantanGelo MI., "Ann. Geof. ", 6, 161 (1953).

(5) Rittmans A., "Zeit. f. Vulk. ", 15, 8 (1933).

(b) Bachelet F., Ferretti-Sfonzini M. e Ippolito F., "Nuovo Cim.», 8, 851 (1951).

(i) v. Buttlar H. ind Houtermans F. G., "Geoch. et Cosmoch. Acta ", 2, 43 (1951). 